\title{
Coexistence of Gait Disturbances and Chorea in Experimental Huntington's Disease
}

\author{
João Casaca-Carreira, ${ }^{1,2}$ Yasin Temel, ${ }^{1,2,3}$ Marloes van Zelst, ${ }^{1}$ and Ali Jahanshahi ${ }^{1,2,3}$ \\ ${ }^{1}$ Department of Neuroscience, Maastricht University Medical Center, 6229 ER Maastricht, Netherlands \\ ${ }^{2}$ European Graduate School of Neuroscience (EURON), 6229 ER Maastricht, Netherlands \\ ${ }^{3}$ Department of Neurosurgery, Maastricht University Medical Center, 6229 ER Maastricht, Netherlands
}

Correspondence should be addressed to João Casaca-Carreira; j.casacacarreira@maastrichtuniversity.nl and Ali Jahanshahi; a.jahanshahianvar@maastrichtuniversity.nl

Received 1 March 2015; Revised 6 April 2015; Accepted 17 April 2015

Academic Editor: Barbara Picconi

Copyright ( 2015 João Casaca-Carreira et al. This is an open access article distributed under the Creative Commons Attribution License, which permits unrestricted use, distribution, and reproduction in any medium, provided the original work is properly cited.

\begin{abstract}
Huntington's disease (HD) is an autosomal dominant neurodegenerative disease caused by an expanded CAG repeat. The clinical features are progressive motor dysfunction, cognitive deterioration, and psychiatric disturbances. Unpredictable choreic movements, among the most characteristic hallmarks, may contribute to gait disturbances and loss of balance in HD individuals. In this study, we aimed to investigate and characterize the gait abnormalities and choreic movements in a transgenic rat model of HD (tgHD). TgHD presents typical neuropathological, neurophysiological, and behavioral aspects mimicking some of the key features of human HD and is the only described experimental model for HD that exhibits choreiform movements. We used the Catwalk, with emphasis on static and dynamic gait parameters, to test the hypothesis that at symptomatic age ( 9 months) the dynamic measures of gait in HD are altered and coexist with choreiform movements. Our results showed that the dynamic parameters seem to be more affected than static parameters at this age in tgHD rats. The number of steps and step cycles and swing speed of the paws were increased in tgHD rat in comparison to wild-type controls. Our study demonstrates that gait abnormalities coexist with chorea rather than being caused by it. These symptoms may originate from distinct networks in the basal ganglia and downstream connections.
\end{abstract}

\section{Introduction}

Huntington's disease (HD) is an autosomal dominant neurodegenerative disease caused by an expanded CAG repeat on the short arm of chromosome 4 [1]. The clinical features are progressive motor dysfunction, cognitive deterioration, and psychiatric disturbances that begin around midlife [2]. However, subtle functional deficits occur years prior to clinical diagnosis [2]. In the early stages of the disease, gait may become unstable [3]. Moreover, slight motor abnormalities, like finger tapping, tongue protrusion, and tandem gait, in premanifest HD (gene carriers not yet demonstrating motor symptoms and functional decline), are associated with an early onset of the disease and smaller striatal volumes [4].

The motor symptoms are among the most characteristic hallmarks, which makes chorea a defining symptom of the disease, known as Chorea or Huntington's chorea. Greek word for dance, chorea, is defined as an unpredictable, rapid, involuntary, nonrepetitive movement affecting the face, trunk, and/or limbs [5]. Besides, gait is another aspect of motor function that shows remarkable abnormalities in HD individuals, ranging from lateral or side-to-side deviations and imbalance to difficulties with turning and initiation of gait in some cases. Shorter step length, decreased walking speed, and increased stride time have also been reported in HD patients [6-8]. It has been hypothesized that choreic movements cause gait disturbances and loss of balance in HD individuals [9]. For instance, the augmented gait such as stride-to-stride variability observed in HD is suggested to be due to the excessive choreic movements $[6,8,10]$. In addition, chorea appears to influence factors that contribute to falls in HD. Particularly, clinical scores of chorea were positively associated with the range of angular trunk motion in both mediolateral and anterioposterior directions in HD 


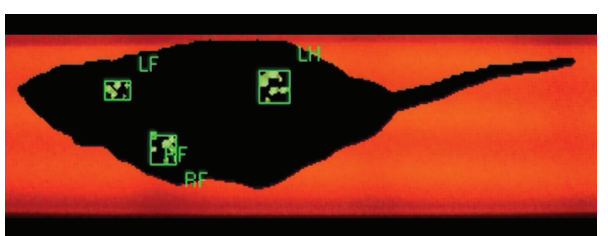

(a)

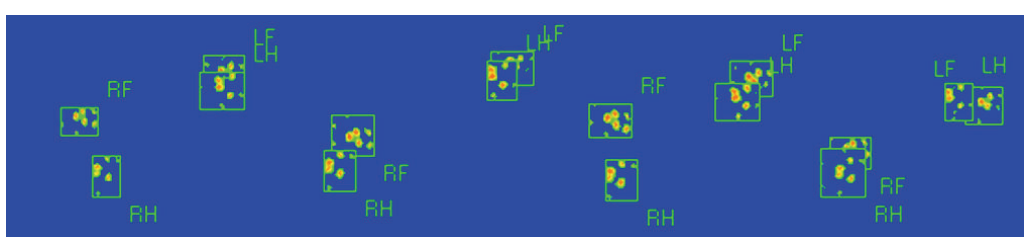

(b)

FiguRE 1: Screen shots represent the Catwalk gait analysis method. (a) A rat, running on the platform. (b) Representation of paw prints in the analysis software. RF: right front paw; LF: left front paw; RH: right hind paw; LH: left hind paw.

individuals [3]. Nonetheless, gait and balance abnormalities do not seem to be purely caused by chorea, regarding the fact that distinct neural networks are shown to underlie these symptoms [11-16]. For instance, clinical data suggest that dysfunction of the basal ganglia cueing mechanism results in impaired locomotion timing in HD. This was illustrated by difficulties in ability to regulate rhythm, an increased variability of stepping rates, and difficulties in achieving synchronized footsteps [8].

To address whether gait is "physically" affected by chorea in HD, we investigated gait and chorea in quadrupedal animal, where balance can be independently accessed without being affected by other symptoms such as chorea.

The discovery of the HD gene in 1993 [1] facilitated the development of several genetically modified rodent models that mimic the onset and progression of the disease. Among those, transgenic rat model of HD ( $\operatorname{tgHD}$ ) has widely been used [17] and presents typical neuropathological, neurophysiological, and behavioral features resembling the human condition $[18,19]$. The tgHD is the only described experimental model of HD exhibiting choreiform movements $[9,17,18,20]$. The onset of hyperkinetic movements is at the ages of 6-8 months, which might differ from one colony to another, and its severity increases with disease progression [9]. In tgHD rats gait abnormalities have been assessed in presymptomatic stages ( 2,3 , and 4 months of age) showing a hyperkinetic profile with increased swing and locomotor speed and decreased paw contact [21]. Following the presymptomatic phase of the disease, hyperkinetic profile changes to hypokinesia in the symptomatic/late phase [17-19, 21]. In this study, we analyzed the static and dynamic parameters during the early symptomatic phase of the disease in tgHD rats. As it was reported before [21], the dynamic parameters tend to be more affected in the early phase of the disease. Here, we decided to analyze also the static parameters, since these parameters are likely to be affected at later ages when the hypokinetic profile of the disease begins to appear.

Automated and quantitative gait analysis method Catwalk [22] was initially used in animal models of spinal cord injury. To date, it is a well-established method to assess gait abnormalities in several models of neurological disorders [21, $23,24]$. The Catwalk provides both dynamics, for example, inter- and intralimb coordination, weight bearing, duration of swing, and stance phases and static parameters of gait, like print length, stride length, base of support, and so forth, in an objective and efficient approach. We used the Catwalk, accessing the static and dynamic gait parameters, to test the hypothesis that gait abnormalities coexist with choreiform movements in HD rather than being caused by it.

\section{Methods}

2.1. Subjects. TgHD rats (Sprague-Dawley background) express a 1962-base-pares rat HD cDNA fragment carrying a 51-CAG repeat under the control of the endogenous rat huntingtin promoter [17]. Homozygous male tgHD rats and wild-type (WT) littermates were housed in pairs under a $12 \mathrm{~h}$ reversed day/night cycle (light from 7 am till $7 \mathrm{pm}$ ), provided with water and food ad libitum. Room temperature $\left(21 \pm 2^{\circ} \mathrm{C}\right)$, humidity $(60 \pm 10 \%)$, and air exchange (16 times per hour) were automatically controlled. The Animal Experiments and Ethics Committee of Maastricht had approved all experimental procedures.

2.2. Experimental Groups. WT $(n=7)$ and $\operatorname{tg} \mathrm{HD}(n=7)$ male rats were bred at the Central Animal Facility of Maastricht University (Maastricht, Netherlands). For genotyping purposes, all the animals were tail tipped at weaning and genotypes determined by PCR.

2.3. Behavioral Testing. All the behavioral tests were performed at the age of approximately 9 months and during the dark phase.

2.3.1. Choreiform Movements. The number of choreiform movements was quantified using the same criteria as described previously $[9,20]$. In brief, all the subjects were videotaped individually in a home cage, with sawdust from their own home cage. The animals had 15 minutes to acclimatize to the cage and room, before the videotaping started. The video recordings were all performed on the same day, during the dark phase and under infrared light. Two independent observers (blinded to the experimental groups) scored the total number of choreiform movements (abrupt, rapid, brief, and unsustained irregular movements of the neck) during an observation period of $20 \mathrm{~min}$. Choreiform movements were not detected in other parts of the body.

2.3.2. Automated Gait Analysis. The gait analysis system Catwalk 7.1 (Noldus IT, Netherlands) consists of an enclosed walkway with a glass plate and a speed video recording camera (Figure 1). Gait performance was assessed and recorded using the Catwalk analysis software. The glass plate was 
TABLE 1: Description of the parameters selected and analyzed from the Catwalk test and respective units of measure.

\begin{tabular}{lll}
\hline Parameter & Description & Unit \\
\hline Base of support (BOS) & Average width between front and hind paws & $\mathrm{mm}$ \\
\hline Stride length & Distance between successive placements of the same paw & $\mathrm{mm}$ \\
\hline Step cycle & $\begin{array}{l}\text { Time between } 2 \text { consecutive contacts with the glass plate of the } \\
\text { same paw (stance }+ \text { swing phase) }\end{array}$ \\
\hline Swing speed & Speed of the paw during the swing phase & $\mathrm{sm} / \mathrm{s}$ \\
\hline Average speed & Distance covered divided by the time used to cross & $\mathrm{mm} / \mathrm{s}$ \\
\hline Cadence & Number of steps per second \\
\hline Number of steps & Number of steps during a run \\
\hline
\end{tabular}

cleaned and dried before testing each subject to minimize the transmission of olfactory clues and prevent animals from stopping to smell or explore something during a run.

One week prior to the first test session, rats were trained to walk down the runway daily [24]. The training and testing were done by a researcher, which was blinded to the genotype of the animals. As experienced elsewhere [21, 24], some of the animals showed reduced mobility or motivation to cross the complete runway within an acceptable timeframe. To overcome this, some strategies have been used, like prior acclimatization [21] or reducing the number of consecutive runways to include in the analysis [24]. In our case, we decided to test the animals until 3 complete runs were achieved. An acceptable run was defined as an uninterrupted walk of the runway. For every parameter, the average of three runs was used for the analysis.

The Catwalk analysis software produces several parameters, and in previous studies the criteria for the selection of these parameters are not always justified. Here, the decision to include these parameters was based on the only study using the Catwalk system in tgHD rats and also on human studies that performed gait analysis (Table 1).

2.4. Statistical Analysis. Individual runs were analyzed by the Catwalk software. Three individual runs from each animal were used to compute mean and standard error of the mean (SEM) for all the Catwalk parameters. A separate analysis was done for every parameter belonging to each paw, unless stated otherwise. Statistical analysis between the 2 experimental groups was done by applying independent sample $t$-test. Interobserver agreement on the animals that showed choreiform movements was assessed using the weighed kappa coefficient. Data are presented as means and standard error of means (SEM). All statistical analyses were performed with SPSS 20.0 version for Windows, with $P$ values smaller than 0.05 considered significant.

\section{Results}

Among the static parameters analyzed, stride length (Figure 2) was augmented in tgHD rats $(P=0.02)$, while the base of support did not show any significant difference between groups (data are not shown).

In the dynamic parameters, tgHD rats showed decreased number of steps $(P=0.033)$ and a trend of increased speed

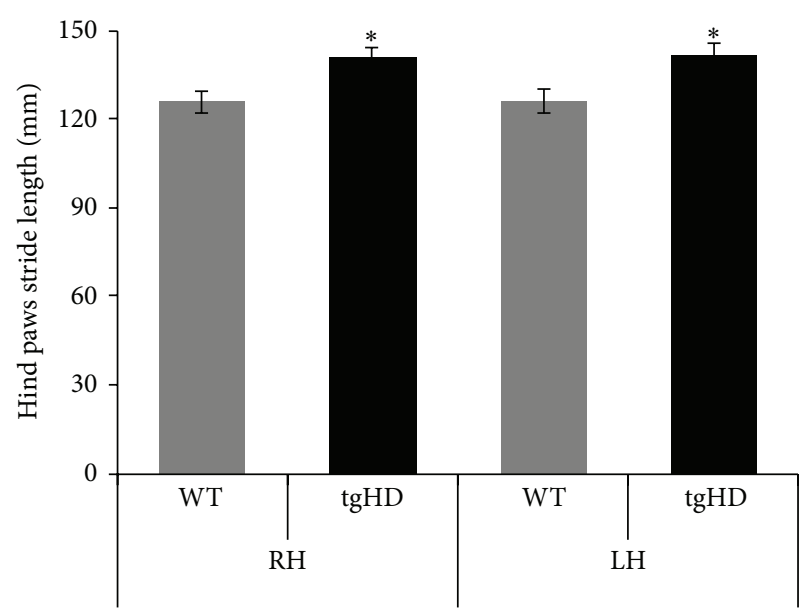

FIGURE 2: Static gait parameter-the graph represents the hind paws stride length in 9-month-old transgenic (tgHD) and wild-type (WT) rats. RH: right hind paw; LH: left hind paw. The values are mean \pm standard error of the mean (SEM) and statistically significant difference $(P<0.05)$ is indicated by an $(*)$.

$(P=0.051)$ when compared with age matched WT animals (Figures 3(a) and 3(b)). In addition, tgHD rats showed a higher swing speed in all paws (Figure $3(\mathrm{c}), P=0.002$ ) and reduced hind paws step cycles $(P=0.033)$ in comparison with age matched WT subjects (Figure $3(\mathrm{~d})$ ). The number of choreiform movements was assessed in both groups at 9 months of age (Figure 4). The WT rats did not show any abnormal movements corresponding to the definition of choreiform movements described in this model. However, on average, the tgHD animals showed 8-9 movements during 20 min of observation.

Of note, kappa value was 0.857 , interpreted as a very good interobserver agreement.

\section{Discussion}

Our behavioral study demonstrated that tgHD rats show gait abnormalities in the early symptomatic phase of the disease. Moreover, the manifestation of locomotor deficits is accompanied by choreiform movements, which are part of the hyperkinetic features of HD. In the adult form of HD, motor disturbances frequently involve hyperkinesia, chorea, 


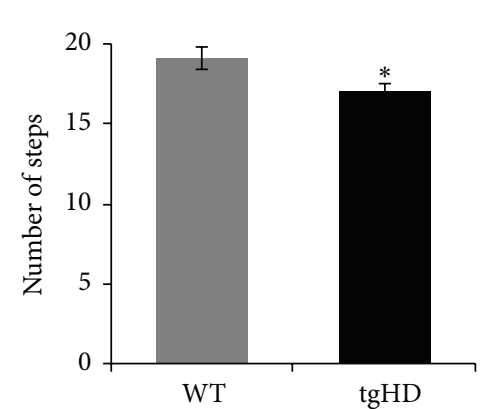

(a)

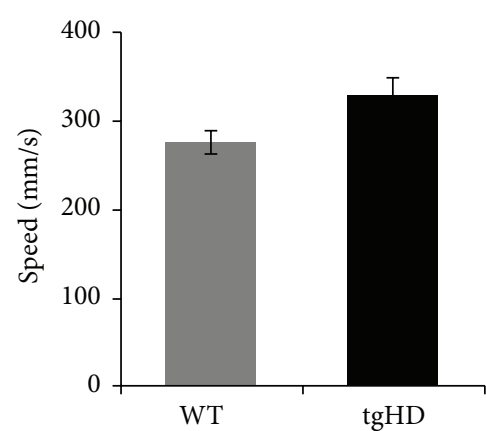

(b)

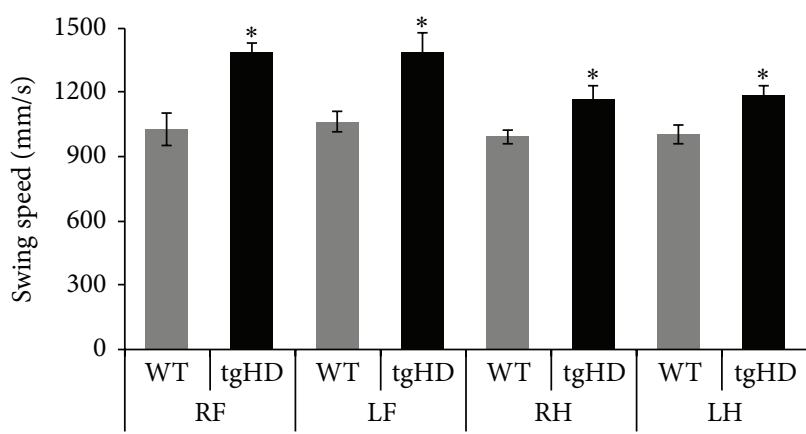

(c)

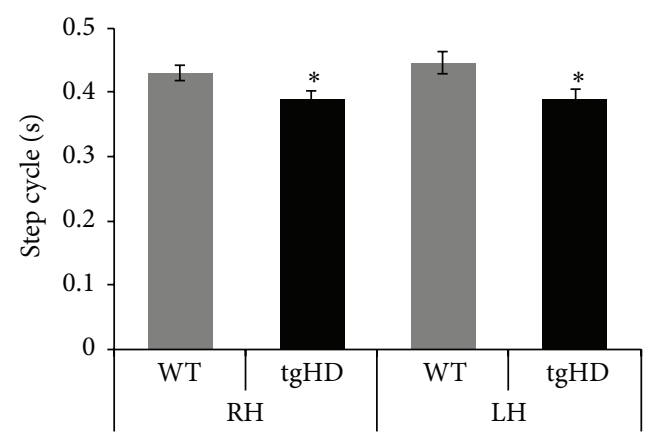

(d)

FIGURE 3: Dynamic gait parameters-(a) the graph shows the average number of steps, comparing transgenic (tgHD) with wild-type (WT) rats. (b) The graph represents the average speed in $\mathrm{mm} / \mathrm{s}$ of the 9 -month-old tgHD versus WT rats. (c) The graph shows the swing speed belongs to 4 paws of tgHD compared with WT rats. (d) The graph shows the step cycles in seconds for the hind paws from the tgHD compared with WT rats. RF: right front paw; LF: left front paw; RH: right hind paw; LH: left hind paw. The values are presented as mean \pm standard error of the mean $(\mathrm{SEM})$ and statistically significant difference $(P<0.05)$ is indicated by an $(*)$.

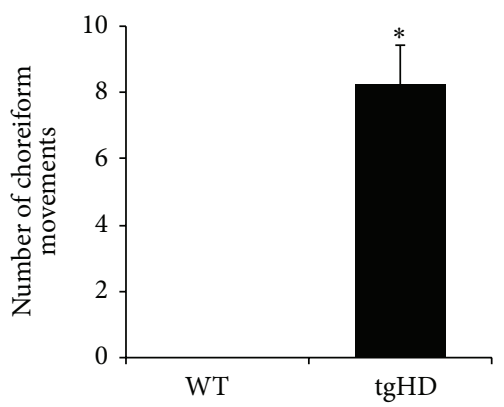

Figure 4: The graph represents the number of choreiform movements scored during 20 minutes. The values are presented as mean \pm standard error of the mean (SEM) and statistically significant difference $(P<0.05)$ is indicated by an $(*)$.

and progressive akinetic/rigid symptoms at more advanced stages [7].

Among the static parameters of gait, base of support, and stride length, only the latter turned out to be significantly increased in the hind paws of the tgHD rats. These results are in line with earlier human studies, showing that stride length is frequently increased in HD subjects when compared to healthy controls [7]. Interestingly, reduction in stride length, which is considered as a hypokinetic feature, can be found at later ages, showing that gait follows the hyperkinetic and hypokinetic course of the disease in HD [7]. Of note, the phenotype of the tgHD rats comprises an early symptomatic phase, characterized by hypermobility and a late disease phase, where hypokinesia is the dominant symptom [18, 19, 25], resembling the human condition.

The fact that the base of support did not differ between the two groups can be explained by the age, at which the rats were evaluated. Due to the known hyperkinetic/hypokinetic profile of this model, we expect hypokinesia to appear later in the progression of the disease's late symptomatic phase. Base of support is by definition the average width between the front and hind paws and is frequently increased to compensate for an instable gait [26] and to increase balance [27], which we expect to occur at later stages of the disease in tgHD rats.

As we hypothesized, the dynamic parameters seem to be more affected at this age than the static parameters in tgHD rats. Our results showed that the number of steps, step cycles and swing speed were increased. All these parameters seem to indicate a hyperkinetic profile in tgHD rats, which is still detectable at 9 months of age. Dynamic parameters can be influenced by velocity [27]; yet in our study this seems to not be the case, since velocity was not statistically different between two groups. Nevertheless, there was a clear trend $(P=0.051)$ on the velocity parameter, where the tgHD group was faster than the wild-types. This trend might be 
interpreted as a sign that the hyperkinetic presymptomatic trait of this model [17-19] is becoming less apparent and the hypokinesia is starting to be the dominant symptom.

Through gait analysis, it was shown that tgHD rats in pre-manifestation phase have a hyperkinetic profile, characterized by increased swing speed, decreased paw contact, and increased speed [21]. This hyperkinetic profile was not detectable on the Rotarod test but only with the Catwalk, which seems to be a more sensitive method for detecting locomotor deficits. Notably, 2- to 5-month-old rats at premanifest stage of HD were examined in this study, but only at 2 months of age an increased speed was detected. According to previous reports $[9,17-20]$, at these "early" ages the tgHD do not fully express the disease profile, while 9-month-old rats were used in our study, which seems to be the onset time of the neurodegeneration phase. Besides establishing the gait assessment, we also replicated the presence of choreiform movements, which so far have been only identified in this rodent model. Such feature makes tgHD a suitable model to study these particular motor symptoms and possible relations with other motor symptoms. On average, we observed 89 choreiform movements of the tgHD rats in a time frame of 20 minutes. The number of choreiform movements that was scored in our experiment was substantially lower than those described earlier $[9,20]$. This might be explained by the age of the animals tested, since the number of choreiform movements tends to increase with age. In our study, the animals were assessed at 9 months of age, the early symptomatic phase, while previously the animals had been assessed in their late symptomatic phase $[9,20]$.

Our results appear to be in line with the human studies, which show that chorea has an influence on gait. However, a more detailed interpretation of the number of choreiform movements observed would not be able to predict a direct effect of chorea on gait. In fact, the probability of overlapping a choreiform movement with the duration of the Catwalk run seems to be unlikely. A direct relation between chorea and gait deficits, decreased balance, and falls has been suggested before [3, 7], whilst others have questioned this relation. In a clinical study, Reynolds and coworkers [10] investigated the gait in $6 \mathrm{HD}$ patients and found out that chorea in HD does not considerably affect the center of gravity during ambulation, despite random and frequent variability during gait cycle. An earlier study [6] reported that, even when chorea was clearly improved with neuroleptics, gait abnormalities were still detectable, mainly variability in swing and stance times. Moreover, in favor of this reasoning is the fact that gait abnormalities were detected at 2 months of age in tgHD [21] when choreiform movements are not yet present. Another argument supporting the hypothesis that gait deficits are not influenced by chorea arises from the quadruped locomotion of the rats, which is traduced in a more stable and wide base of support. Consequently a movement of the head-choreiform movement-would not be enough to provoke an alteration on the number of steps and step cycles and swing speed, as we have demonstrated.

This apparent contradiction hints once more the coexistence of hyperkinetic and hypokinetic disturbances in
$\mathrm{HD}$, possibly due to the involvement of different mechanisms/pathways in the pathology of motor symptoms. Our previous works have shown that (i) the tgHD rats exert a hyperdopaminergic status, expressed in an increased number of dopaminergic cells in the brainstem, accompanied by an enhanced dopamine expression in the striatum [28, 29]; (ii) the choreiform movements can be reduced by the administration of tetrabenazine, a reversible inhibitor of the vesicular monoamine transporter [9], similar to clinical practice. Based on these findings, it can be suggested that the choreiform movements are originated due to hyperactivity of the nigrostriatal dopaminergic system.

On the other hand, gait changes identified in HD can be linked to deficits in striatum, namely, caudate and putamen atrophy [30]. Sequential and preferential loss of different types of striatal projection neurons in HD may explain distinct origins for chorea and gait [11-16]. According to human postmortem studies, the early loss of GABAergic striatosubstantia nigra pars reticulata neurons may cause the hyperactivity of dopaminergic neurons leading to early appearance of chorea. In addition, the loss of striato-globus pallidus externus neurons, which suppress undesirable movements, takes place earlier than striato-globus pallidus internus neurons, which control and promote wanted movements. This suggests that the appearance of akinesia and, consequently, the gait abnormalities have a different anatomical substrate.

In conclusion, we suggest that gait abnormalities in tgHD rats originate from the striatal deficits and possibly impairment of the executive functions. On the other hand, chorea is seemingly caused by dysregulation of the nigrostriatal dopaminergic pathway.

\section{Conflict of Interests}

The authors declare that there is no conflict of interests regarding the publication of this paper.

\section{References}

[1] M. Macdonald, "A novel gene containing a trinucleotide repeat that is expanded and unstable on Huntington's disease chromosomes," Cell, vol. 72, no. 6, pp. 971-983, 1993.

[2] R. I. Scahill, N. Z. Hobbs, M. J. Say et al., "Clinical impairment in premanifest and early Huntington's disease is associated with regionally specific atrophy," Human Brain Mapping, vol. 34, no. 3, pp. 519-529, 2013.

[3] Y. M. A. Grimbergen, M. J. Knol, B. R. Bloem, B. P. H. Kremer, R. A. C. Roos, and M. Munneke, "Falls and gait disturbances in Huntington's disease," Movement Disorders, vol. 23, no. 7, pp. 970-976, 2008.

[4] K. M. Biglan, C. A. Ross, D. R. Langbehn et al., "Motor abnormalities in premanifest persons with Huntington's disease: the PREDICT-HD study," Movement Disorders, vol. 24, no. 12, pp. 1763-1772, 2009.

[5] F. O. Walker, "Huntington's disease," The Lancet, vol. 369, no. 9557, pp. 218-228, 2007.

[6] W. C. Koller and J. Trimble, "The gait abnormality of Huntington's disease," Neurology, vol. 35, no. 10, pp. 1450-1454, 1985.

[7] A. Delval, P. Krystkowiak, J.-L. Blatt et al., "Role of hypokinesia and bradykinesia in gait disturbances in Huntington's disease: 
a biomechanical study," Journal of Neurology, vol. 253, no. 1, pp. 73-80, 2006.

[8] B. Bilney, M. E. Morris, A. Churchyard, E. Chiu, and N. Georgiou-Karistianis, "Evidence for a disorder of locomotor timing in Huntington's disease," Movement Disorders, vol. 20, no. 1, pp. 51-57, 2005.

[9] D. H. Zeef, A. Jahanshahi, R. Vlamings et al., "An experimental model for Huntington's chorea?" Behavioural Brain Research, vol. 262, pp. 31-34, 2014.

[10] N. C. Reynolds Jr., J. B. Myklebust, T. E. Prieto, and B. M. Myklebust, "Analysis of gait abnormalities in Huntington disease," Archives of Physical Medicine and Rehabilitation, vol. 80, no. 1, pp. 59-65, 1999.

[11] S. J. Augood, R. L. M. Faull, and P. C. Emson, "Dopamine D1 and $\mathrm{D} 2$ receptor gene expression in the striatum in Huntington's disease," Annals of Neurology, vol. 42, no. 2, pp. 215-221, 1997.

[12] M. Glass, M. Dragunow, and R. L. M. Faull, “The pattern of neurodegeneration in Huntington's disease: a comparative study of cannabinoid, dopamine, adenosine and GABA(A) receptor alterations in the human basal ganglia in Huntington's disease," Neuroscience, vol. 97, no. 3, pp. 505-519, 2000.

[13] S. Goto, A. Hirano, and R. R. Rojas-Corona, "Immunohistochemical visualization of afferent nerve terminals in human globus pallidus and its alteration in neostriatal neurodegenerative disorders," Acta Neuropathologica, vol. 78, no. 5, pp. 543550, 1989.

[14] E. K. Richfield, K. A. Maguire-Zeiss, C. Cox, J. Gilmore, and P. Voorn, "Reduced expression of preproenkephalin in striatal neurons from Huntington's disease patients," Annals of Neurology, vol. 37, no. 3, pp. 335-343, 1995.

[15] E. K. Richfield, K. A. Maguire-Zeiss, H. E. Vonkeman, and P. Voorn, "Preferential loss of preproenkephalin versus preprotachykinin neurons from the striatum of Huntington's disease patients," Annals of Neurology, vol. 38, no. 6, pp. 852-861, 1995.

[16] E. K. Richfield, C. F. O’Brien, T. Eskin, and I. Shoulson, "Heterogeneous dopamine receptor changes in early and late Huntington's disease," Neuroscience Letters, vol. 132, no. 1, pp. 121-126, 1991.

[17] S. von Horsten, I. Schmitt, H. P. Nguyen et al., "Transgenic rat model of Huntington's disease," Human Molecular Genetics, vol. 12, no. 6, pp. 617-624, 2003.

[18] J. C. Carreira, A. Jahanshahi, D. Zeef et al., "Transgenic rat models of Huntington's disease," in Current Topics in Behavioral Neurosciences, Springer, Berlin, Germany, 2013.

[19] R. Vlamings, D. H. Zeef, M. L. F. Janssen et al., "Lessons learned from the transgenic Huntington's disease rats," Neural Plasticity, vol. 2012, Article ID 682712, 8 pages, 2012.

[20] C. Cao, Y. Temel, A. Blokland et al., "Progressive deterioration of reaction time performance and choreiform symptoms in a new Huntington's disease transgenic ratmodel," Behavioural Brain Research, vol. 170, no. 2, pp. 257-261, 2006.

[21] C. Vandeputte, J.-M. Taymans, C. Casteels et al., "Automated quantitative gait analysis in animal models of movement disorders," BMC Neuroscience, vol. 11, article 92, 2010.

[22] F. P. T. Hamers, A. J. Lankhorst, T. J. Van Laar, W. B. Veldhuis, and W. H. Gispen, "Automated quantitative gait analysis during overground locomotion in the rat: Its application to spinal cord contusion and transection injuries," Journal of Neurotrauma, vol. 18, no. 2, pp. 187-201, 2001.
[23] L. Yu-Taeger, E. Petrasch-Parwez, A. P. Osmand et al., "A novel BACHD transgenic rat exhibits characteristic neuropathological features of Huntington disease," Journal of Neuroscience, vol. 32, no. 44, pp. 15426-15438, 2012.

[24] J. E. Westin, M. L. F. Janssen, T. N. Sager, and Y. Temel, "Automated gait analysis in bilateral Parkinsonian rats and the role of L-DOPA therapy," Behavioural Brain Research, vol. 226, no. 2, pp. 519-528, 2012.

[25] D. H. Zeef, R. Vlamings, L. W. Lim et al., "Motor and non-motor behaviour in experimental Huntington's disease," Behavioural Brain Research, vol. 226, no. 2, pp. 435-439, 2012.

[26] F. P. T. Hamers, G. C. Koopmans, and E. A. J. Joosten, "CatWalkassisted gait analysis in the assessment of spinal cord injury," Journal of Neurotrauma, vol. 23, no. 3-4, pp. 537-548, 2006.

[27] R. Vlamings, V. Visser-Vandewalle, G. Koopmans et al., "High frequency stimulation of the subthalamic nucleus improves speed of locomotion but impairs forelimb movement in Parkinsonian rats," Neuroscience, vol. 148, no. 3, pp. 815-823, 2007.

[28] A. Jahanshahi, R. Vlamings, W. M. C. van Roon-Mom et al., "Changes in brainstem serotonergic and dopaminergic cell populations in experimental and clinical Huntington's disease," Neuroscience, vol. 238, pp. 71-81, 2013.

[29] A. Jahanshahi, R. Vlamings, A. H. Kaya et al., "Hyperdopaminergic status in experimental Huntington disease," Journal of Neuropathology \& Experimental Neurology, vol. 69, no. 9, pp. 910-917, 2010.

[30] J. Collett, P. Esser, H. Khalil et al., "Insights into gait disorders: walking variability using phase plot analysis, Huntington's disease," Gait \& Posture, vol. 40, no. 4, pp. 694-700, 2014. 


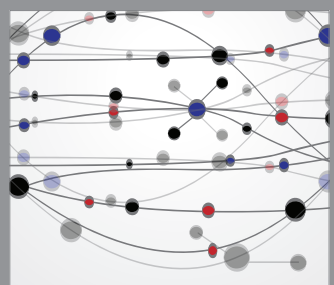

The Scientific World Journal
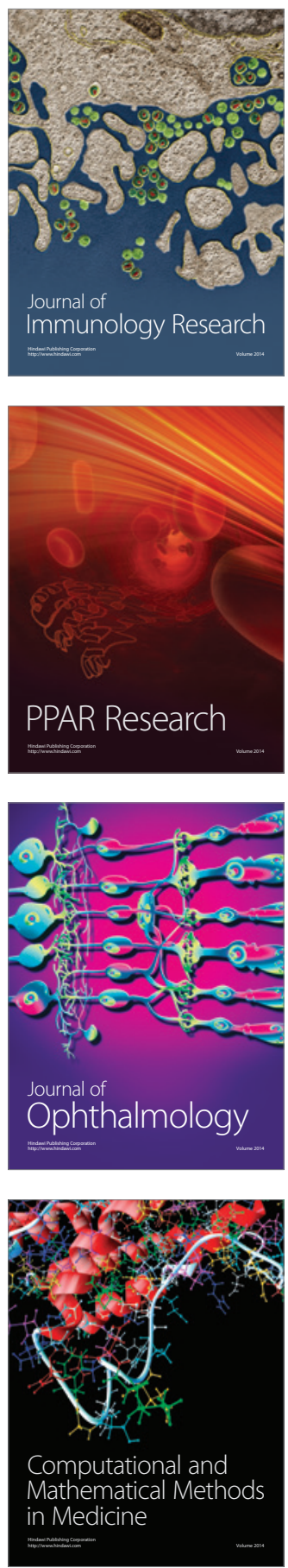

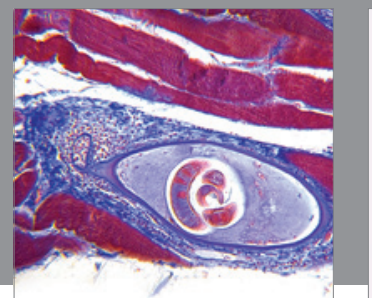

Gastroenterology

Research and Practice
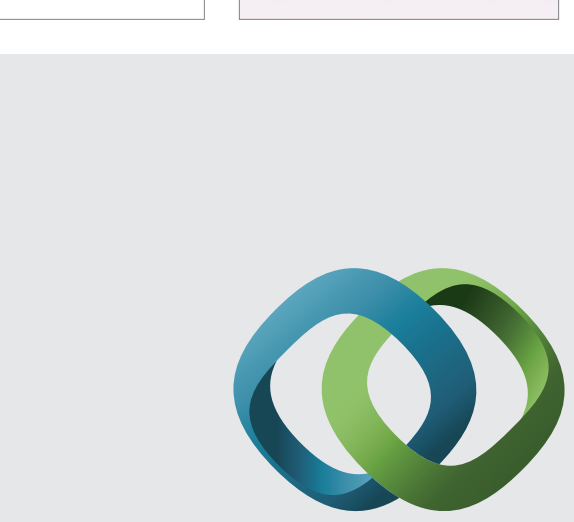

\section{Hindawi}

Submit your manuscripts at

http://www.hindawi.com
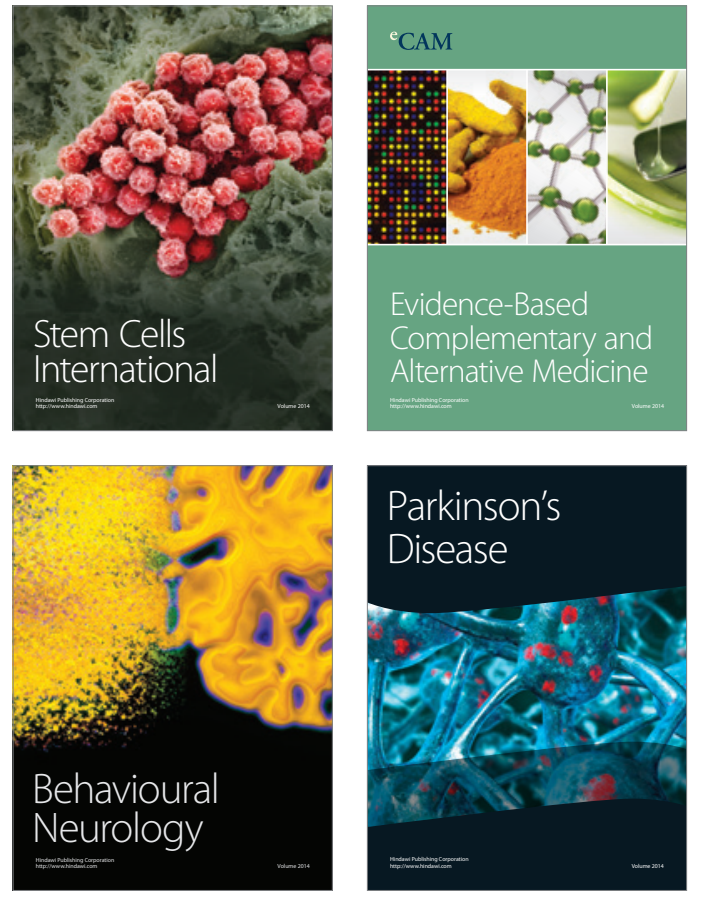
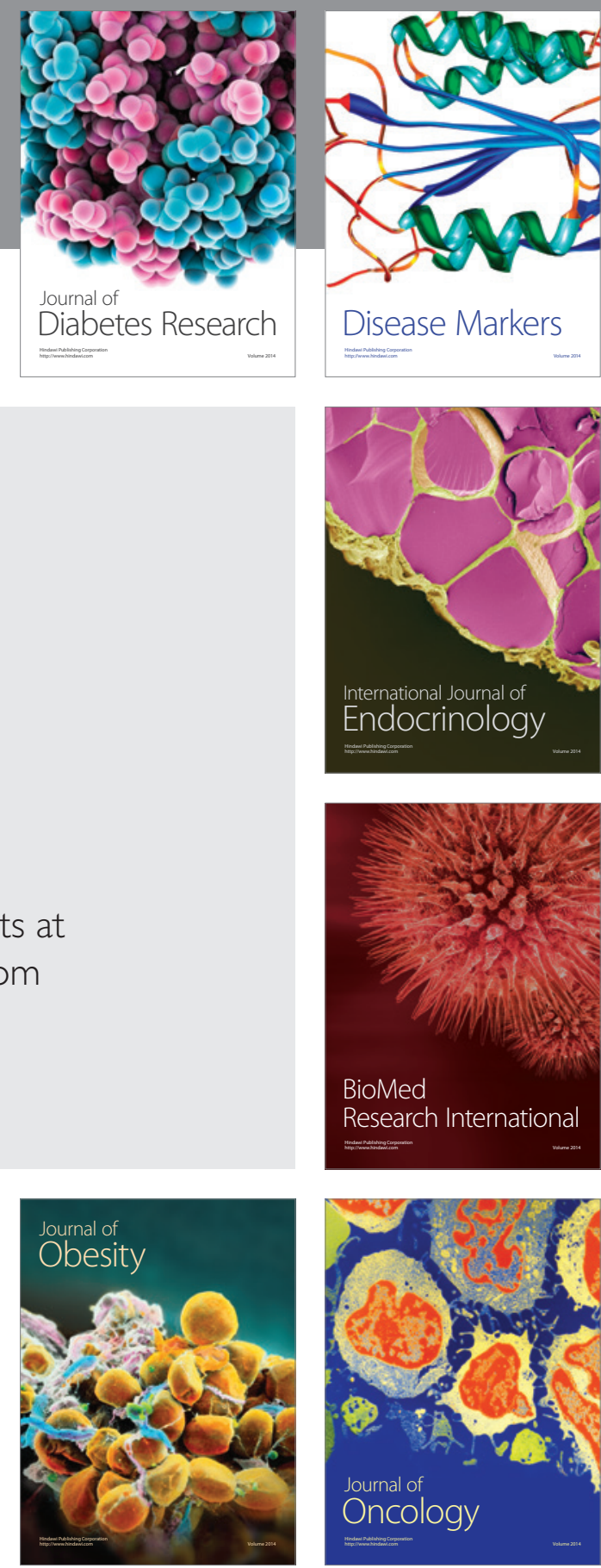

Disease Markers
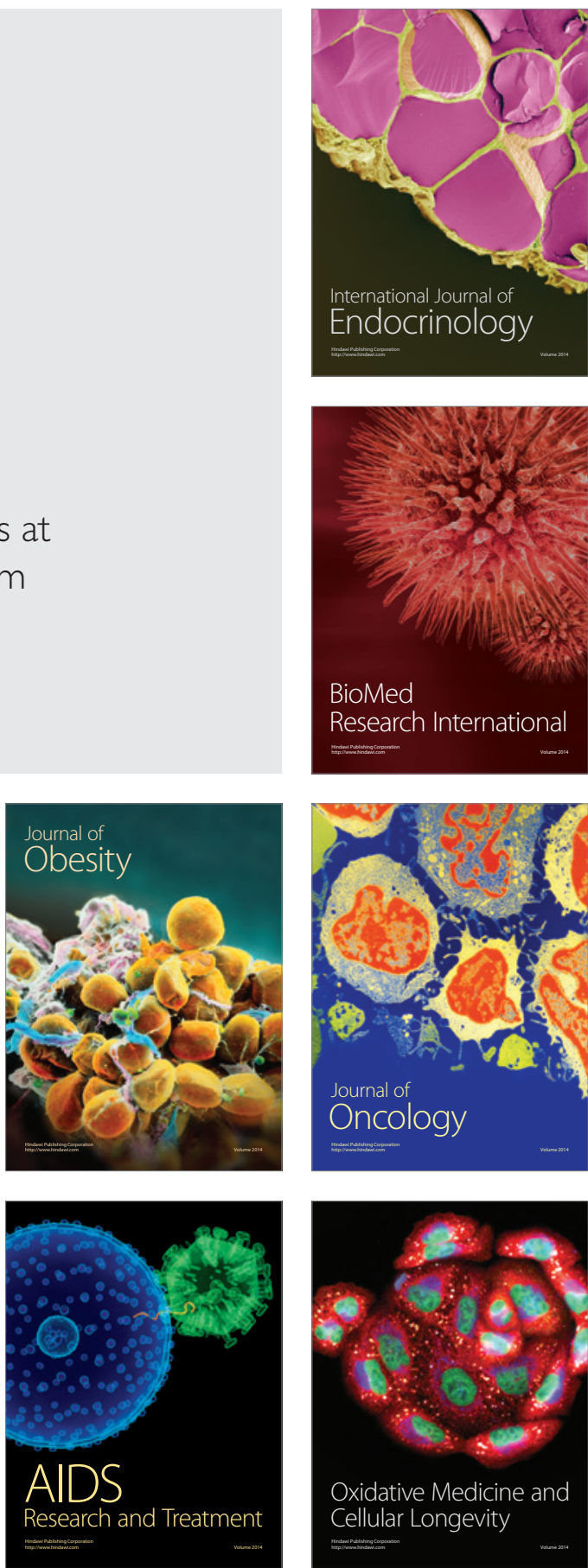